\title{
Analysis and Design of Timing Recovery Schemes for DMT Systems over Indoor Power-Line Channels
}

\author{
José Antonio Cortés, Luis Díez, Eduardo Martos-Naya, Francisco Javier Cañete, and \\ José Tomás Entrambasaguas \\ Departamento de Ingeniería de Comunicaciones, Escuela Técnica Superior de Ingeniería de Telecomunicación,
Universidad de Málaga, 29071 Málaga, Spain
}

Received 31 October 2006; Accepted 23 March 2007

Recommended by Moisés Vidal Ribeiro

Discrete multitone (DMT) modulation is a suitable technique to cope with main impairments of broadband indoor power-line channels: spectral selectivity and cyclic time variations. Due to the high-density constellations employed to achieve the required bit-rates, synchronization issues became an important concern in these scenarios. This paper analyzes the performance of a conventional DMT timing recovery scheme designed for linear time-invariant (LTI) channels when employed over indoor power lines. The influence of the channel cyclic short-term variations and the sampling jitter on the system performance is assessed. Bit-rate degradation due to timing errors is evaluated in a set of measured channels. It is shown that this synchronization mechanism limits the system performance in many residential channels. Two improvements are proposed to avoid this end: a new phase error estimator that takes into account the short-term changes in the channel response, and the introduction of notch filters in the timing recovery loop. Simulations confirm that the new scheme eliminates the bit-rate loss in most situations.

Copyright (c) 2007 José Antonio Cortés et al. This is an open access article distributed under the Creative Commons Attribution License, which permits unrestricted use, distribution, and reproduction in any medium, provided the original work is properly cited.

\section{INTRODUCTION}

The increasing demand for home networking capabilities, along with the recent provisioning of triple-pay services (internet, video, and telephony) by digital subscriber line operators, has generated considerable interest in high-speed indoor power-line communications. Applications range from audio/video distribution and traditional local area networking to the connection of computers and entertainment equipment to the network access gateway. The study presented in this paper concentrates on this scenario. However, it may be also useful for outdoor power-line applications because, since the user modem is located in an indoor network, it also experiences the characteristics of the indoor channels.

The available bandwidth for broadband indoor powerline communications (PLC) extends up to $30 \mathrm{MHz}$ [1]. Channels are frequency- and time-selective, with significant differences between the locations of a specific site. The frequency response introduces remarkably amplitude and phase distortion, with deep notches that appear in a priori unknown positions, and the noise is strongly colored $[2,3]$. Time variations have a twofold origin: long-term changes caused by the connection and disconnection of electrical de- vices, with a time frame in the order of minutes or hours [3]; and short-term changes due to the dependence of the electrical devices impedance and emitted noise on the instantaneous mains voltage. The latter causes the channel frequency response to exhibit cyclic short-term variations and the noise to present cyclostationary components, both synchronous with the mains [4].

DMT is an appropriate solution to cope with the aforementioned impairments. The division of the available bandwidth into smaller subbands allows to comply with electromagnetic compatibility (EMC) regulations and to exploit the spectral resources even when they are sparse. Similarly, timevarying channels can be fully exploited by adapting the constellation transmitted in each carrier to the instantaneous channel conditions.

Synchronization becomes an important concern when large spectral efficiencies are needed. Timing errors cause attenuation and phase rotation of the symbols, intercarrier interference (ICI) and, if not properly corrected, may result in a severe drift of the symbol timing. Nowadays, the most common procedure to accomplish synchronization is by means of a fixed frequency sampling and a digital phase-locked loop (PLL) $[5,6]$. This system performs two main tasks: timing 
recovery and timing correction. The former estimates the phase error of the received symbols and, by means of a feedback loop, computes the correction to be applied by the latter. When dense constellations are to be employed, the timing correction is carried out with an interpolator filter $[5,7]$.

Synchronization issues in scenarios with high signal-tonoise ratio (SNR) carriers have been extensively studied in digital subscriber loop (DSL) applications [5-7]. However, it has been shown that when the same strategies are employed in power-line channels, their performance can be seriously degraded [8]. This inferior performance has a twofold origin: the short-term variations of the channel response and the jitter of the sampling process [8]. Uncompensated cyclic shortterm variations of the channel, with harmonics of $50 \mathrm{~Hz}-$ $60 \mathrm{~Hz}$ (depending on the mains signal frequency), mislead the estimation of the sampling error. The periodical bias in the phase error estimates can be reduced by narrowing the loop bandwidth, but this also reduces the loop's capacity to track the sampling jitter [8]. The effect of random period instabilities has been extensively studied in the downconversion of orthogonal frequency-division multiplexing (OFDM) signals [9]. In the analog-to-digital conversion, its influence has been always neglected due to the relatively narrowband signals involved (as in asymmetric DSL). However, when sampling broadband signals, the relative magnitude of the jitter with respect to the sampling period increases and its effects cannot be neglected $[8,10]$.

In this paper, a new timing recovery scheme for indoor PLC is proposed. To this end, the shortcomings of the conventional strategies designed for DMT systems that operate in LTI channels are firstly revisited [8]. This analysis suggests two direct improvements: to design a phase error estimator that takes into account the magnitude of the cyclic short-term changes in the channel response, and to modify the loop response so that higher attenuation is provided to the harmonics of the cyclic channel variations. Performance gains and computational complexity of both alternatives are presented and discussed.

The rest of the paper is organized as follows. In Section 2, models employed for the channel, the timing jitter, and the DMT receiver are described. Bit-rates obtained with the conventional synchronization scheme in indoor power-line scenarios are given in Section 3. The proposed phase estimator and loop filter are defined in Section 4. Performance improvement and computational load increment of the new scheme are also assessed in this section. Finally, main conclusions drawn from the presented results are summarized in Section 5.

\section{SYSTEM MODEL}

\subsection{Channel model}

Provided that the working state of the electrical devices remains unaltered, the channel can be modeled as a linear periodically time-variant (LPTV) system plus a cyclostationary Gaussian noise term (neglecting asynchronous impulse noise). Fortunately, the delay spread of these channels is

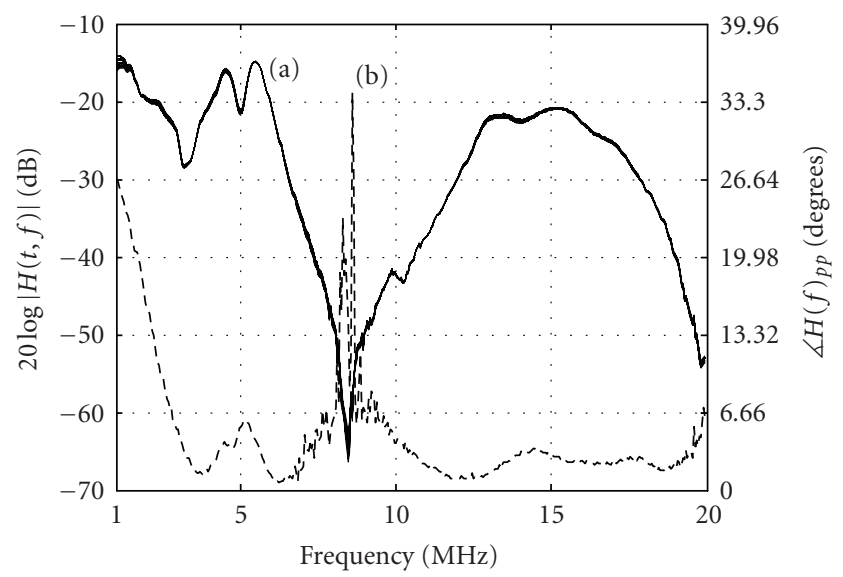

FIgURE 1: (a) Superimposed values of the amplitude channel response along the mains cycle. (b) Peak excursion of the channel phase response along the mains cycle.

much smaller than their coherence time, that is, the channels are underspread, and a slow-variation approach can be assumed [4].

Simulations presented in this paper have been carried out over a set of 24 channels measured in the frequency band from $1 \mathrm{MHz}$ to $20 \mathrm{MHz}$ in two indoor scenarios: 12 in an apartment of about $80 \mathrm{~m}^{2}$ and 12 in a detached house of about $300 \mathrm{~m}^{2}$. A statistical characterization of the frequency responses and the noise instantaneous power spectral densities (IPSD) can be found in [4]. The effect of a bandpass coupling circuit that serves as an antialiasing filter and protects the receiver from the mains signal is included in all cases. Throughout this work, averaged performance values computed using the overall set of channels are always preceded by qualitative results obtained in one of them. A representative apartment channel has been selected to this end. In Figure 1(a), the modulus of the frequency response, $H(t, f)$, along the mains cycle has been superimposed (left axis). Figure 1(b) shows information about phase changes of the frequency response. However, this time only the peak excursion of the phase along a cycle time, defined as

$$
\varangle H(f)_{p p}=\max _{t}[\varangle H(t, f)]-\min _{t}[\varangle H(t, f)],
$$

where $t \in\left[0, T_{0}\right)$ and $T_{0}=20$ milliseconds, has been depicted (right axis).

As observed, there are no significant amplitude changes, and discarding the $4 \mathrm{~dB}$ that occur in the vicinity of the notch, the maximum variation is about $2.5 \mathrm{~dB}$ around $1 \mathrm{MHz}$. On the contrary, remarkable phase changes occur in the $1 \mathrm{MHz}$ to $3 \mathrm{MHz}$ band and in the neighborhood of $5 \mathrm{MHz}$ and $9 \mathrm{MHz}$.

Noise in this channel is shown in Figure 2, where the values of the IPSD, $S_{U}(t, f)$, measured along a mains cycle have been depicted. It is worth noting that differences exceeding $20 \mathrm{~dB}$ do occur between $2 \mathrm{MHz}$ and $3 \mathrm{MHz}$ and about $15 \mathrm{~dB}$ around $5 \mathrm{MHz}$. 


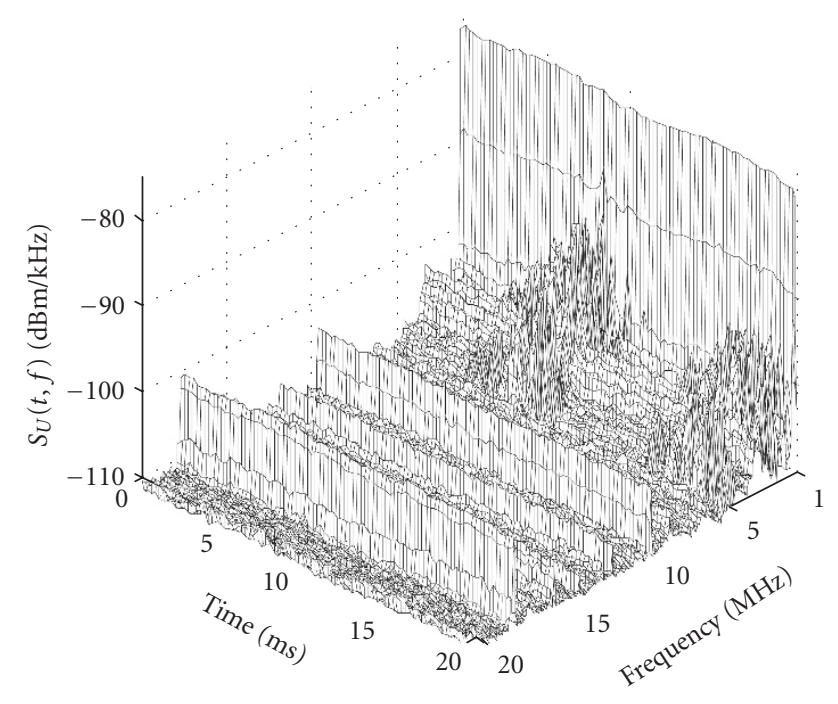

FIGURE 2: Cyclostationary noise instantaneous PSD in the example channel.

\subsection{Analog-to-digital conversion model}

The sampling instants of an analog-to-digital conversion process experience two types of deviation from their nominal values. The first is a systematic effect due to the frequency inaccuracy of the clock that drives the analog-todigital converter (ADC). The influence of this phenomenon in the performance of DMT systems has been widely studied [5-7], and will not be considered in this work unless otherwise stated. The second is a random deviation with two components: one due to the fluctuations of the ADC sampling clock period, the so-called oscillator jitter, and another due to the uncertainty in the sampling instant introduced by the sample-and-hold (S\&H) circuit of the ADC, the so-called aperture jitter [11].

The signal generated by an actual sinusoidal oscillator without amplitude instabilities has the form

$$
s(t)=A \cos \left(2 \pi f_{S} t+\phi_{o}(t)\right),
$$

where $\phi_{o}(t)$ is the phase noise that models the random fluctuations caused by the noise sources of the circuit that generates the oscillation [12]. Due to the phase noise, the significant instants of the signal, for example, zero crossings, experience a time deviation from their nominal values. This is the so-called timing jitter or simply jitter, $\tau_{o}(t)$, whose relation with the phase noise can be generally approximated by $\tau_{o}(t) \approx-\phi_{o}(t) / 2 \pi f_{S}$.

Random period instabilities can be characterized in the frequency domain by means of the phase noise spectrum, $S_{\phi_{o}}(f)$ [12]. For distant frequencies from the carrier, this magnitude is related with the so-called single-sideband (SSB) phase noise spectrum

$$
\mathcal{L}(f)=10 \log _{10}\left(\frac{S_{s}(f)}{A^{2}}\right) \approx 10 \log _{10}\left(\frac{S_{\phi_{o}}(f)}{2}\right)(\mathrm{dBc} / \mathrm{Hz}),
$$

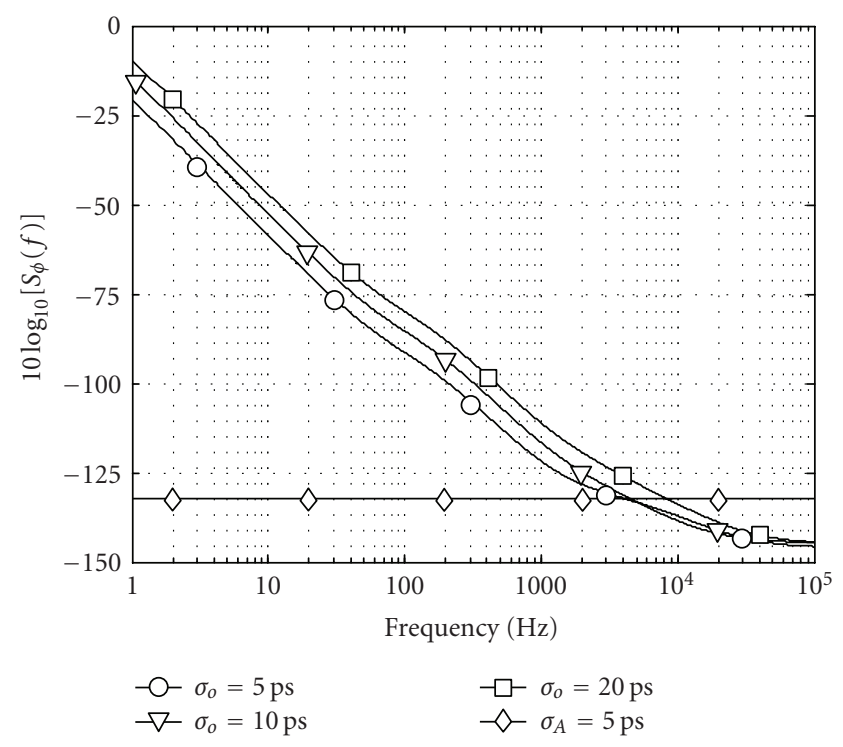

FIGURE 3: Oscillator and aperture phase noise spectra.

where $S_{s}(f)$ is the PSD of the signal in (2) and $\mathrm{dBc} / \mathrm{Hz}$ stands for $\mathrm{dB}$ below the carrier power in a $1 \mathrm{~Hz}$ bandwidth. $\mathcal{L}(f)$ is a very popular magnitude because it can be measured in a quite simple way with a spectrum analyzer. In the time domain, the most employed magnitude is the integrated jitter, $\sigma_{o}$, computed as

$$
\sigma_{o}^{2}=\frac{1}{\left(2 \pi f_{S}\right)^{2}} \int_{f_{L}}^{f_{H}} S_{\phi_{o}}(f) d f
$$

where $f_{L}$ is usually fixed to $10 \mathrm{~Hz}$ and $f_{H}$ to $20 \mathrm{MHz}$.

Phase noise is accurately characterized by means of a power-law model $[12,13]$. It approximates $S_{\phi_{o}}(f)$ by a piecewise linear function whose slopes are in the range from $-40 \mathrm{~dB} /$ decade to $0 \mathrm{~dB} /$ decade with $10 \mathrm{~dB} /$ decade steps. Jitter values employed in this work have been generated by filtering a Gaussian white noise with a cascade of first- and secondorder transfer functions that approximate the different slopes of the phase noise spectrum. Figure 3 shows the curves corresponding to three $100 \mathrm{MHz}$ state-of-the-art oscillators with integrated jitter values of 20 picoseconds, 10 picoseconds and 5 picosecond. This model is sometimes simplified, for example, when modeling the phase noise of the oscillators employed in the downconversion of OFDM signals, and only the $-20 \mathrm{~dB} /$ decade slope is considered [9]. This leads to a Lorentzian shape for $\mathcal{L}(f)$ and to an analytically tractable problem [14]. However, it produces excessively optimistic results when used to model the timing jitter of the ADC driving clock.

Even if an ideal oscillator could be used, actual sampling instant would fluctuate due to the aperture jitter, $\tau_{A}(t)$. The usual method to model these random instabilities is by means of the aperture phase noise PSD, which is assumed to 


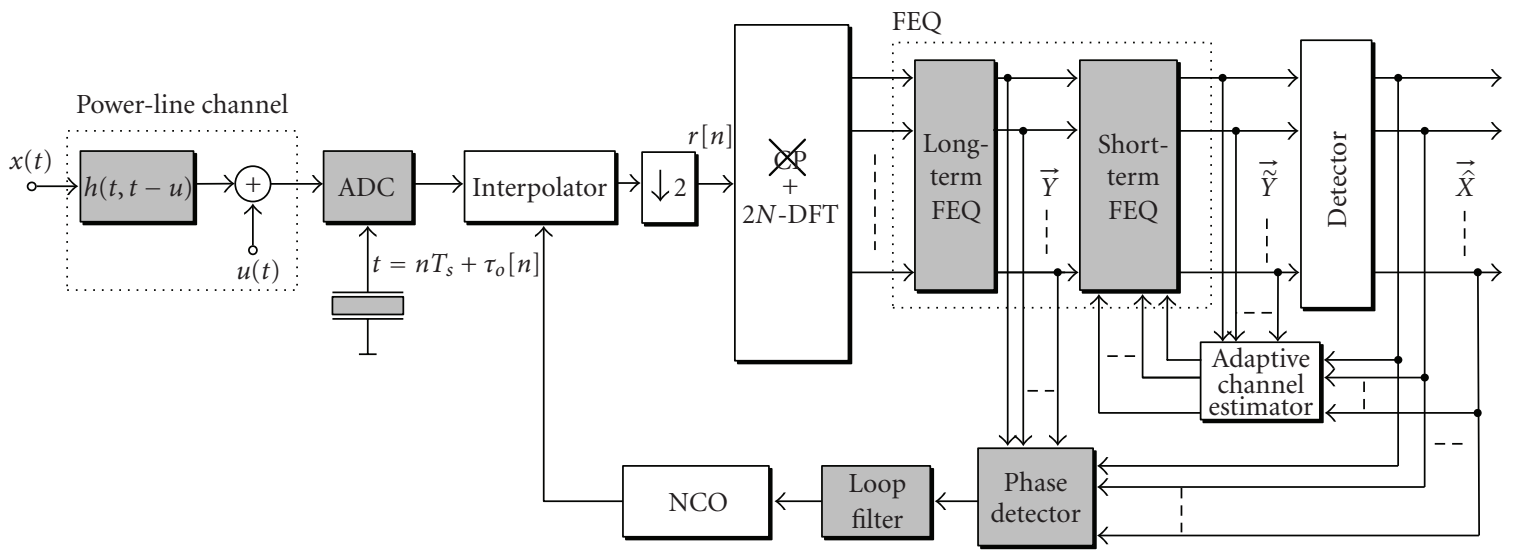

FIGURE 4: Simplified scheme of the DMT receiver.

have a Lorentzian shape [11]

$$
S_{\phi_{A}}(f)=2 \pi \frac{\sigma_{A}^{2} f_{S}^{2}}{f_{S}^{2} / 4+f^{2}},
$$

where $\sigma_{A}^{2}$ is the aperture jitter RMS value. Figure 3 depicts the aperture phase noise PSD of a state-of-the-art 12bit, $100 \mathrm{MHz}$ sampling frequency analog-to-digital converter with 5 picosecond of RMS aperture jitter. As seen, in the frequency band of interest, it is essentially flat.

Provided that the circuit has a well-designed layout, both types of instabilities can be assumed to be independent. Hence, their corresponding PSDs can be summed to obtain the overall jitter, which from now on will be referred to as ADC jitter.

\subsection{DMT receiver model}

The simplified block diagram of an $N$ carrier DMT receiver with an all-digital synchronization scheme is shown in Figure 4. The incoming signal is oversampled with an unsynchronized clock. Timing error correction is carried out in the time domain by means of an interpolator. The oversampling is performed to reduce the typical performance degradation experienced by interpolator filters in the vicinity of half the Nyquist frequency. By designing interpolator filters according to the technique described in [7], it can be ensured that signal distortion is essentially due to the timing recovery errors.

As observed, the frequency equalizer (FEQ) that follows the discrete fourier transform (DFT) is performed in two stages. The reason is that according to the statistics of the Doppler spread bandwidth shown in [4], the taps of a onestage FEQ should be adapted at a rate comparable to that of the synchronization system. This may cause interaction between both adaptive systems and their eventual divergence [15]. Hence, a long-term FEQ (LFEQ) is firstly used to compensate for the long-term changes in the channel response. Since these changes occur at a rate much slower than the symbol rate, the information needed for timing recovery is taken from the output of this stage. Afterwards, a short- term FEQ (SFEQ) follows the short-term variations of the channel response with respect to its long-term value. Since channels considered in this work do not present long-term changes, the LFEQ compensates for the time-average channel response over the mains cycle.

The timing recovery scheme follows the conventional digital phase-locked loop (PLL) structure: an estimator of the phase errors due to the uncorrected timing errors, a loop filter, and a numerically controlled oscillator (NCO).

\section{CONVENTIONAL TIMING RECOVERY SCHEME}

In a first instance, this section describes the conventional timing recovery mechanism employed in DMT systems that operate in LTI channels. To this end, the expressions of the most common phase estimator and loop filter employed in time-invariant channels are firstly presented. Afterwards, the effect of the cyclic changes in the channel over the outputs of the phase estimator and the loop filter is identified and the performance of the overall timing recovery scheme is assessed.

\subsection{Description}

The timing error in the signal $r[n]$ (see Figure 4) varies from sample to sample due to the uncorrected jitter and frequency offset. Assuming an LTI channel and following a similar approach to the one in $[6,7]$, it can be shown that in the absence of ISI, the expression of the $m$ th input symbol to the receiver DFT can be expressed as ${ }^{1}$

$$
r_{m}[n]=\sum_{k=-(N-1)}^{N} H_{k} X_{m, k} e^{j(\pi / N) k\left(n+\tau_{m}[n]\right)}+u_{m}[n],
$$

where $0 \leq n \leq 2 N-1, X_{m, k}$ denotes the $m$ th complex value transmitted in carrier $k, H_{k}$ is the channel frequency response

\footnotetext{
${ }^{1}$ For simplicity, the effect of the cyclic prefix is not considered because it does not change the essence of the analysis.
} 
for carrier $k, \tau_{m}[n]$ is the uncorrected sampling error for the $n$th sample of the $m$ th symbol, and $u_{m}[n]$ is the channel noise term.

The $k$ th output of the DFT of $r_{m}[n]$ can then be expressed as

$$
\begin{aligned}
R_{m, k}= & \frac{1}{2 N} \sum_{n=0}^{2 N-1} r_{m}[n] e^{-j(\pi / N) k n} \\
= & \frac{1}{2 N} \sum_{n=0}^{2 N-1} \sum_{z=-(N-1)}^{N} H_{z} X_{m, z} e^{j(\pi / N)(z-k) n} e^{j(\pi / N) z \tau_{m}[n]} \\
& +\frac{1}{2 N} \sum_{n=0}^{2 N-1} u_{m}[n] e^{-j(\pi / N) k n} \\
= & \frac{1}{2 N} H_{k} X_{m, k} \sum_{n=0}^{2 N-1} e^{j(\pi / N) k \tau_{m}[n]} \\
& +\frac{1}{2 N} \sum_{z=-(N-1)}^{N} \sum_{z=0}^{2 N-1} H_{z} X_{m, z} e^{j(\pi / N)(z-k) n} e^{j(\pi / N) z \tau_{m}[n]} \\
& +U_{m, k},
\end{aligned}
$$

and the $k$ th output of the conventional one-tap FEQ, $Y_{m, k}$, is given by

$$
\begin{aligned}
Y_{m, k}= & \frac{1}{2 N} X_{m, k} \sum_{n=0}^{2 N-1} e^{j(\pi / N) k \tau_{m}[n]} \\
& +\frac{1}{2 N} \mathrm{FEQ}_{k} \sum_{\substack{z=-(N-1) \\
z \neq k}}^{N} \sum_{n=0}^{2 N-1} H_{z} X_{m, z} e^{j(\pi / N)(z-k) n} e^{j(\pi / N) z \tau_{m}[n]} \\
& +U_{m, k} \mathrm{FEQ}_{k},
\end{aligned}
$$

where $\mathrm{FEQ}_{k}=H_{k}^{-1}$ is the $k$ th tap of a zero-forcing FEQ. The first term in (8) is the desired symbol, which is attenuated and phase shifted, the second term represents the ICI. Provided that the timing error variation along a DMT symbol is small, the attenuation of the desired symbol and the ICI term can be neglected. Hence, $Y_{m, k}$ can be approximated by

$$
Y_{m, k} \approx X_{m, k} e^{j(\pi / N) k \theta_{m}}+U_{m, k} \mathrm{FEQ}_{k},
$$

where $\theta_{m}$ is the phase error caused by the timing errors which occurred during the $m$ th symbol,

$$
\theta_{m} \approx \frac{1}{2 N} \sum_{n=0}^{2 N-1} \tau_{m}[n]
$$

To verify the validity of the approximation in (9), the signalto-distortion ratio (SDR) at the detector input, defined as

$$
\mathrm{SDR}_{k}=\frac{E\left[\left|X_{m, k}\right|^{2}\right]}{E\left[\left|X_{m, k}-Y_{m, k}\right|^{2}\right]}
$$

has been computed using the expression for $Y_{m, k}$ given in (8) and the one in (9). For simplicity, a DMT system with

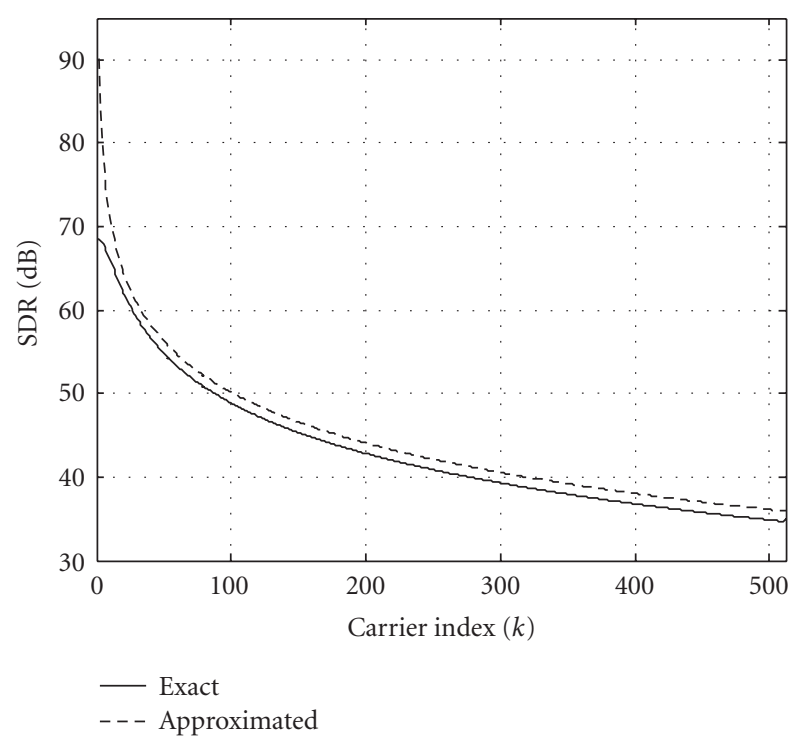

FIGURE 5: SDR values computed by taking into account the ICI and the desired symbol attenuation (exact) or only the phase shift (approximated).

512 carriers working in a noiseless flat channel and impaired by an uncorrected sampling offset of $10 \mathrm{ppm}$ is considered. Results are depicted in Figure 5. As seen, the phase shift is the dominating term in nearly all carriers. The difference between both curves is lower than $1.2 \mathrm{~dB}$ except for the low carriers region. However, it should be taken into account that most of the carriers in this latter zone cannot be used because they fall within the reject band of the coupling circuit used to protect the receiver from the mains. Moreover, all the carriers in which the difference between both curves is higher than $2 \mathrm{~dB}$ experience SDR values higher than $60 \mathrm{~dB}$. Hence, the channel noise, and not the ICI, will be the limiting term in these carriers.

The phase detector can estimate $\theta_{m}$ based on the decided symbols (decision-directed) or using one or more predefined carriers designated as pilots. Pilot-based schemes do not seem to be appropriate for indoor power-line environments due to their larger variance and to the unknown position of the channel frequency response notches. Hence, the former approach has been selected in this work. The maximum likelihood (ML) is probably the most widely employed estimator for this purpose. Assuming correct decisions and Gaussian noise, its expression can be approximated by [5]

$$
\widehat{\theta_{m}} \approx \frac{N}{\pi} \frac{\sum_{k \in K}\left(\operatorname{Im}\left[Y_{m, k} \hat{X}_{m, k}^{*}\right] k /\left[\left|\mathrm{FEQ}_{k}\right|^{2} \sigma_{U_{k}}^{2}\right]\right)}{\sum_{k \in K} k^{2} \mathrm{SNR}_{k}},
$$

where $\operatorname{Im}[\cdot]$ denotes the imaginary part, $\hat{X}_{m, k}^{*}$ is the complex conjugate of the detector output, $\mathrm{SNR}_{k}$ is the signal-to-noise ratio experienced by carrier $k, K$ is the set of carrier indexes utilized in the estimation, and $\sigma_{U_{k}}^{2}$ is the noise power in the band of carrier $k$. 
The output of the phase detector is fed to a loop filter, whose transfer function

$$
L(z)=\alpha+\frac{\beta}{1-z^{-1}}
$$

is selected so that a second-order-type II PLL results [15]. The output of this filter is supplied to the NCO, which computes the timing adjustment to be applied to each sample of the next received symbol.

In order to analyze the performance of the ML estimator calculated according to (12) in an LPTV channel with cyclostationary noise, it is convenient to express the symbol index, $m$ in terms of the channel and noise IPSD period. Denoting $L=T_{0} / T_{\mathrm{DMT}}$, where $T_{\mathrm{DMT}}$ is the DMT symbol period and $T_{0}$ is the mains period, $m=c L+\ell$, where $0 \leq \ell \leq L-1$ and $c$ is the cycle index. Assuming that the slow-variation approach holds [4], the output of the LFEQ at the frequency of carrier $k$ during the $\ell$ th interval of the $c$ th cycle $Y_{\ell, k}^{c}$ can be expressed as

$$
Y_{\ell, k}^{c} \approx X_{\ell, k}^{c}\left|H_{\ell, k}\right| e^{j \varangle H_{\ell, k}} e^{j(\pi / N) k \theta_{\ell}^{c}} \mathrm{LFEQ}_{k}+U_{\ell, k}^{c} \mathrm{LFEQ}_{k},
$$

where $H_{\ell, k}$ denotes the frequency response of the channel at the frequency of carrier $k$ during the $\ell$ th symbol and $U_{\ell, k}^{c}$ is the noise value in carrier $k$ at the output of the $\ell$ th DFT performed in the $c$ th cycle.

Since the LFEQ only compensates for the time-averaged value of the frequency response, $\mathrm{LFEQ}_{k}=\left\langle H_{\ell, k}\right\rangle^{-1}$, where $\langle\cdot\rangle$ denotes averaging over the variable $\ell$, the output of the phase detector in (7) would be misled by the cyclic changes of the channel.

\subsection{Performance}

This subsection analyzes the performance of the above synchronization scheme when employed over the 24 measured channels. Qualitative results over the example channel shown in Figures 1 and 2 are firstly presented. Throughout the work, the following system parameters apply unless otherwise stated. A DMT with 512 carriers distributed in the frequency band up to $25 \mathrm{MHz}$ is employed. However, only carriers with indexes $22 \leq k \leq 409$, that is, in the band from approximately $1 \mathrm{MHz}$ to $20 \mathrm{MHz}$, are finally used. The sampling frequency is fixed to $1 / T_{s}=100 \mathrm{MHz}$. The cyclic prefix length $c p$ has been fixed to 226 samples at $1 /\left(2 T_{s}\right)$, which ensures that the power of ISI and ICI due to the spectral distortion of the channel will be much lower than the channel noise level. This cyclic prefix length also makes $T_{0} / T_{\mathrm{DMT}}$ an integer value, which simplifies the subsequent analysis. An ideal equalization is accomplished, that is, the LFEQ and SFEQ are provided with the actual frequency response values. The bitloading process is performed with the objective of maximizing the bit-rate subject to an instantaneous bit error probability of $P_{e}=10^{-5}$ and a transmitter PSD constraint of $-20 \mathrm{dBm} / \mathrm{kHz}$. BPSK and square QAM constellations with a maximum of 16 bits/symbol are employed. A system margin of $6 \mathrm{~dB}$ is employed. The loop filter is configured for the overall PLL response to be critically dumped.

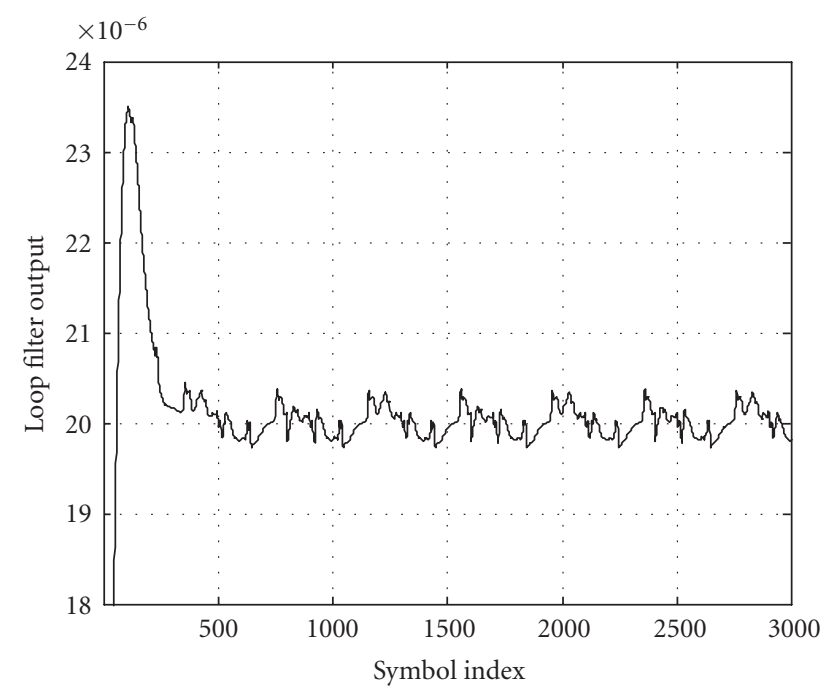

Figure 6: Loop filter output in the channel of Figures 1 and 2 for a frequency error of $20 \mathrm{ppm}$.

Figure 6 shows the loop filter output when the only nonideal effect introduced by the ADC is a frequency offset of $20 \mathrm{ppm}$. The equivalent noise bandwidth of the loop is set to $510 \mathrm{~Hz}$. As seen, periodical components caused by the LPTV nature of the channel are manifest at the filter output. They can be diminished by reducing the loop bandwidth. However, this leads to longer convergence times and, therefore, to a reduction in the capacity of the loop to follow timing fluctuations like the ones shown in Figure 3.

To illustrate the reduced tracking capacity of narrowband loops, Figure 7 depicts the loop output for a $3 \mathrm{~Hz}$ sinusoidal input jitter. An LTI channel has been used. As shown, there are no significant amplitude differences for the three considered bandwidths. Moreover, for $10 \mathrm{~Hz}$ and $30 \mathrm{~Hz}$, the output amplitude is even greater than for $125 \mathrm{~Hz}$ due to the unavoidable peaking that appears in the frequency response of a critically dumped second-order-type II PLL [15]. On the other hand, it is worth noting the remarkably delay increment that occurs for $10 \mathrm{~Hz}$. When the loop bandwidth is large, the group delay is high in frequencies, where the $S_{\phi_{0}}(f)$ of an actual jitter is very small. As the loop bandwidth is reduced, the delay becomes larger in the low frequency region, where the jitter has its most significant components.

Performance of the timing recovery procedure is characterized in terms of the SDR at the detector input and the achievable bit-rate. For small timing errors, the SDR experienced by carrier $k$ in the $\ell$ th interval of each cycle can be expressed as

$$
\begin{aligned}
\operatorname{SDR}_{\ell, k} & =\frac{E\left[\left|X_{\ell, k}^{c}\right|^{2}\right]}{E\left[\left|X_{\ell, k}^{c}-X_{\ell, k}^{c} e^{j(\pi / N) k \theta_{\ell}^{c}}\right|^{2}\right]} \\
& =\frac{1}{E\left[\left|1-e^{j(\pi / N) k \theta_{\ell}^{c}}\right|^{2}\right]} .
\end{aligned}
$$

Estimated values of the SDR experienced by the last used carrier $(k=409)$ in the example channel as a function of the 


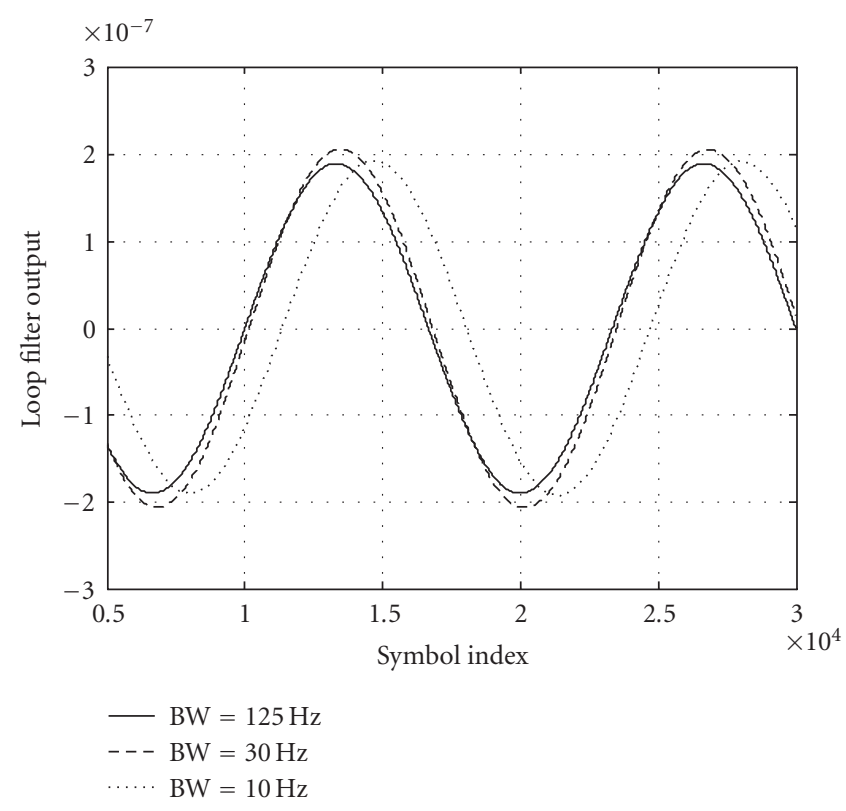

FIgURE 7: Loop filter output for a $3 \mathrm{~Hz}$ sinusoidal input jitter in an LTI channel.

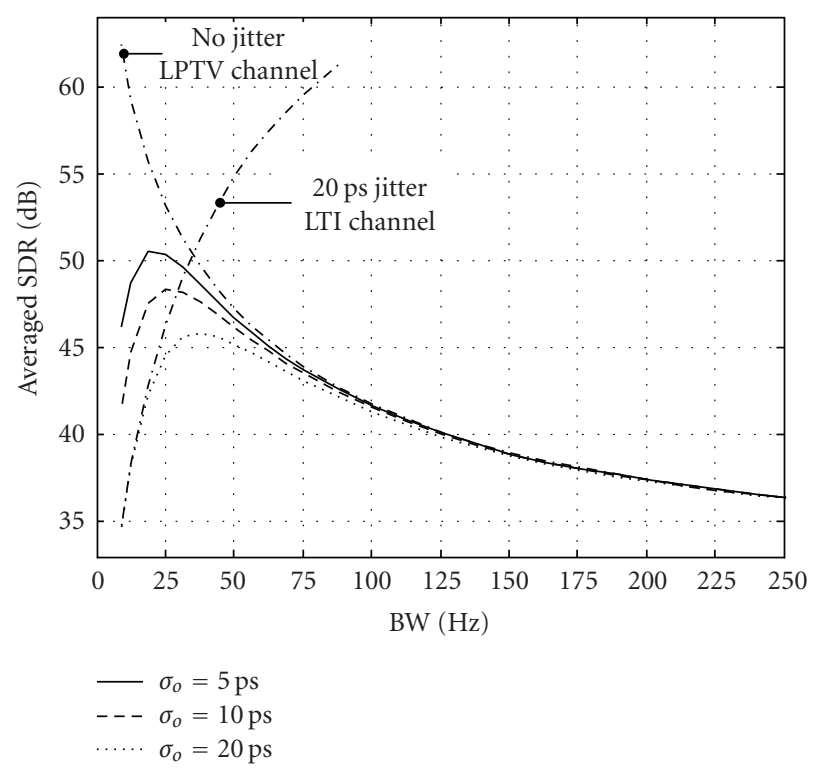

FIGURE 8: SDR in the last used carrier in different situations.

loop bandwidth have been obtained by means of simulations. Results obtained with the ADC jitters depicted in Figure 3 are shown in Figure 8. Although curves are labelled according to the oscillator integrated jitter values, a 5 picosecond aperture jitter is also included in all cases. No frequency offset exists. Two additional curves have been depicted to highlight the individual effect of the channel cyclic variations and the ADC jitter in the system performance. One of them shows the SDR values obtained when the 20 picosecond oscillator is employed in an LTI channel obtained by means of a time averaging of the LPTV one displayed in Figure 1. The other de-

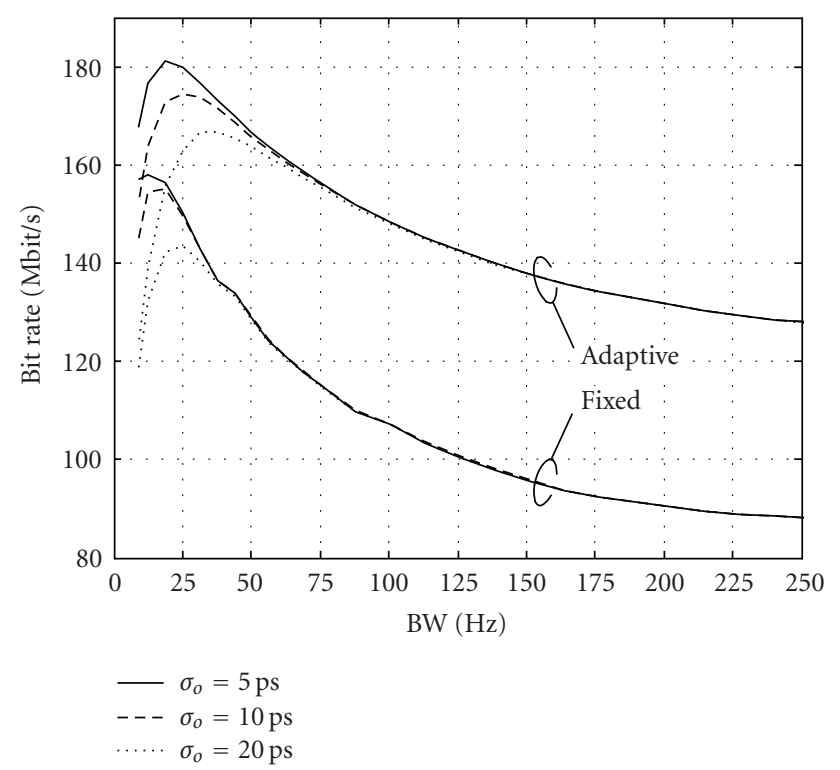

FIGURE 9: Bit-rates estimated from SNDR values and a lookup table.

picts the SDR values obtained when the time-variant channel shown in Figures 1 and 2 is employed but no jitter is introduced in the ADC process.

Curves in Figure 8 illustrate a clear tradeoff in the selection of the loop bandwidth. For larger loop bandwidths, the SDR is low because phase error estimates are strongly misled by the cyclic short-term variations of the frequency response (the cyclostationary noise has much less influence). As the loop bandwidth is reduced, the SDR increases because channel time variations are attenuated. This process continues until the loop is not able to follow the ADC jitter. From this point on, distortion caused by this phenomenon becomes the dominating term and the SDR degrades very fast.

The ultimate system performance parameter is the achievable bit-rate. However, its exact computation under the considered circumstances is a difficult task. Moreover, values calculated in this way may not reflect the bit-rate attained by an actual system. A practical procedure to determine the bit load of each carrier in a real receiver would estimate the signal-to-noise-and-distortion ratio (SNDR). According to the usual assumption of an additive Gaussian noise and distortion, the most appropriate constellation for each carrier is obtained by means of a predefined lookup table. Figure 9 depicts the bit-rate values obtained with this procedure in the example channel. Two different modulation strategies have been considered: fixed and adaptive. In the fixed one, the same constellation is employed in each carrier throughout the mains period, while in the adaptive one it is adjusted according to the instantaneous conditions to make the most of the periodically varying behavior of the channel.

It can be observed that differences between the bit-rates shown in Figure 9 experience a considerably increment when the loop bandwidth is enlarged. For instance, when the 5 picosecond case is considered, the bit-rate gain obtained with the adaptive system for a $19 \mathrm{~Hz}$ bandwidth is about $16 \%$ 
greater than the one provided by a fixed scheme. When a $160 \mathrm{~Hz}$ bandwidth is employed, this gain goes up to about $45 \%$. The reason is that the wider the loop bandwidth is, the larger the magnitude of the channel time variations at the output of the phase detector is, and consequently the greater the dispersion in SDR values is. Figure 9 also shows the great sensitivity of the bit-rate with respect to the bandwidth, especially in the jitter-limited region. Thus, a small bandwidth reduction over the optimum values for the 5 picosecond case may reduce the performance of both the adaptive and the nonadaptive systems, to nearly the ones of the 20 picosecond case. Hence, the investment in a better oscillator is not always productive.

So far, qualitative effects of the jitter and short-time variations in the timing recovery mechanism have been presented. Statistical values of the performance degradation computed over the 24 channels referred to in Section 2.1 are now given. To this end, the bit-rate loss experienced in both scenarios with each oscillator and modulation strategy is computed. Bit-rates obtained in each channel under perfect synchronization conditions are taken as reference for the comparison. The bandwidth employed in all the channels is fixed for each modulation strategy and oscillator. These bandwidths values are computed by averaging the optimum bandwidths of all the channels in the selected configuration. Results are shown in Table 1. As seen, considerable performance degradation occurs in the apartment channels, especially when the constellation remains fixed throughout the mains cycle. Bit-rate losses are smaller in the detached house due to the inherently worse characteristics of these channels, which are established over longer and more branched links than in the apartment.

Exact values of the performance degradation depend on the set of available constellations, the objective bit error probability, and the system margin [8]. However, the only way to reduce the remarkable bit-rate losses experienced in the apartment channels is to modify the timing recovery scheme.

\section{PROPOSED TIMING RECOVERY SCHEME}

The conventional synchronization scheme admits two direct improvements when used in indoor power-line scenarios. Firstly, the phase detector can be matched to the particularities of the problem. This can be accomplished by taking into account the magnitude of the channel time variations before combining the estimates of the phase error obtained in the different carriers. Secondly, the loop filter can be modified to achieve higher attenuation at the harmonics of the mains frequency. This could be done by means of a higher-order loop. However, due to the periodical nature of the estimation bias, the introduction of notch filters is a more suitable solution.

\subsection{Proposed phase error estimator}

Since the probability density function of the channel shortterm variations is not precisely known, a weighted leastsquares (LS) estimator is proposed. Let us denote by $\phi_{\ell, k}^{c}$ the
TABle 1: Average bit-rate loss (\%) in each scenario when the optimum loop bandwidth (on average) is employed in all the channels.

\begin{tabular}{l|ccc}
\hline Scenario & $\sigma_{o}=20 \mathrm{ps}$ & $\sigma_{o}=10 \mathrm{ps}$ & $\sigma_{o}=5 \mathrm{ps}$ \\
\hline Apartment (adaptive) & 13.9 & 10.9 & 8.4 \\
Apartment (fixed) & 22.9 & 20.4 & 17.5 \\
Detached house (adaptive) & 3.2 & 2.1 & 1.3 \\
Detached house (fixed) & 6.6 & 5.3 & 4.2 \\
\hline
\end{tabular}

phase error measured in carrier $k$ during the $\ell$ th symbol of the $c$ th cycle, computed according to

$$
\phi_{\ell, k}^{c}=\tan ^{-1}\left(\frac{\operatorname{Im}\left[Y_{\ell, k}^{c} \hat{X}_{\ell, k}^{c^{*}}\right]}{\operatorname{Re}\left[Y_{\ell, k}^{c} \hat{X}_{\ell, k}^{c^{*}}\right]}\right)=\frac{\pi}{N} k \theta_{\ell}^{c}+\varangle \tilde{H}_{\ell, k}+\varphi_{\ell, k}^{c},
$$

where $\hat{X}_{\ell, k}^{c}$ is the $\ell$ th decided symbol of the $c$ th cycle in carrier $k, Y_{\ell, k}^{c}$ is the output value of the LFEQ given in (14), $\varphi_{\ell, k}^{c}$ is the phase noise due to the additive channel noise $U_{\ell, k}^{c}$, and whose power can be approximated by $\sigma_{\varphi_{\ell, k}}^{2} \approx 1 /\left(2 \mathrm{SNR}_{\ell, k}\right)$, provided that $\mathrm{SNR}_{\ell, k} \gg 1$ [16]. $\varangle \tilde{H}_{\ell, k}$ is the difference between the channel phase and the LFEQ phase experienced by the $\ell$ th received symbol in carrier $k$.

The weighted LS estimator of $\theta_{\ell}^{c}$ is selected according to

$$
\begin{aligned}
\hat{\theta}_{\ell}^{c} & =\arg \min _{\tilde{\theta}_{\ell}^{c}}\left\{\sum_{k \in K} \frac{\left|\phi_{\ell, k}^{c}-(\pi / N) k \tilde{\theta}_{\ell}^{c}\right|^{2}}{\left(\varangle \tilde{H}_{\ell, k}^{2}+\sigma_{\varphi_{\ell, k}}^{2}\right)}\right\} \\
& =\frac{N}{\pi} \frac{\sum_{k \in K}\left(k \phi_{\ell, k}^{c} /\left(\varangle \tilde{H}_{\ell, k}^{2}+\sigma_{\varphi_{\ell, k}}^{2}\right)\right)}{\sum_{k \in K}\left(k^{2} /\left(\varangle \tilde{H}_{\ell, k}^{2}+\sigma_{\varphi_{\ell, k}}^{2}\right)\right)} .
\end{aligned}
$$

The selection of the carriers to be employed in the phase estimation is a difficult task, as the mean-squared error (MSE) in (18) reveals

$$
\begin{aligned}
\operatorname{MSE}_{\ell}^{c}= & E\left[\left|\hat{\theta}_{\ell}^{c}-\theta_{\ell}^{c}\right|^{2}\right] \\
= & \frac{(N / \pi)^{2}}{\left[\sum_{k \in K}\left(k^{2} /\left(\varangle \tilde{H}_{\ell, k}^{2}+\sigma_{\varphi_{\ell, k}}^{2}\right)\right)\right]^{2}} \\
& \times\left[\left(\sum_{k \in K} \frac{k \varangle \tilde{H}_{\ell, k}}{\left(\varangle \tilde{H}_{\ell, k}^{2}+\sigma_{\varphi_{\ell, k}}^{2}\right)}\right)^{2}+\sum_{k \in K} \frac{k^{2} \sigma_{\varphi_{\ell, k}}^{2}}{\left(\varangle \tilde{H}_{\ell, k}^{2}+\sigma_{\varphi, k}^{2}\right)}\right. \\
& +2 \theta_{\ell}^{c}\left(\sum_{k \in K} \frac{k \varangle \tilde{H}_{\ell, k}}{\left(\varangle \tilde{H}_{\ell, k}^{2}+\sigma_{\varphi_{\ell, k}}^{2}\right)}\right) \\
& \left.\times\left(\sum_{k \in K} \frac{k^{2}}{\left(\varangle \tilde{H}_{\ell, k}^{2}+\sigma_{\varphi_{\ell, k}}^{2}\right)}\right)\right] .
\end{aligned}
$$

The influence of the channel phase variations and the noise in (18) is evident. However, the first and second terms in the square bracket also highlight the importance of the carrier index, since a given channel variation is more harmful in carriers with higher indexes. Similarly, the third term shows that the estimation error depends on the magnitude to be estimated, $\theta_{\ell}^{c}$, which, in turns is also determined by the loop response.

The computation of (17) involves two main difficulties. Firstly, it requires $K+1$ divisions, or alternatively the storage of the $L \cdot K$ values of $\left(\varangle \tilde{H}_{\ell, k}^{2}+\sigma_{\varphi_{\ell, k}}^{2}\right)^{-1}$. This excessively 


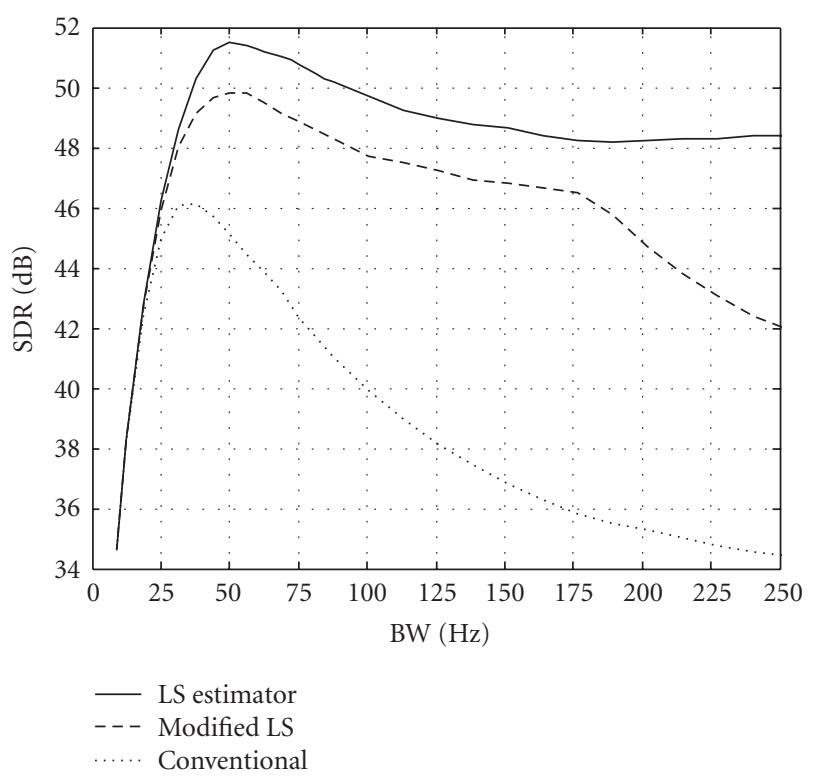

FIGURE 10: SDR values of the proposed and conventional phase error estimators.

high complexity can be reduced by replacing the instantaneous values of $\varangle \tilde{H}_{\ell, k}^{2}+\sigma_{\varphi_{\ell, k}}^{2}$ by their time-averaged values. Secondly, these time-averaged values are still unknown and must be estimated. In this work, an exponential averaging of $\phi_{\ell, k}^{c}$ is employed to perform the estimation. The resulting LS estimator will be referred to as modified LS from now on. Figure 10 compares the SDR values experienced by the last carrier in the example channel when using the LS estimator in (17) and the modified LS. The conventional estimator in (12) is included as a reference. A 20 picosecond oscillator jitter is used in all cases. As expected, there are no SDR differences in the region in which distortion is limited by the jitter. On the contrary, the proposed estimators provide considerable gains in the region where the channel variations limit the performance. In addition, it is worth noting that the SDR becomes less sensitive to the loop bandwidth. The steady-state computational load of the modified LS estimator is equal to that of the conventional one and, as shown in Figure 10, it performs less than $2 \mathrm{~dB}$ worse than the LS in a quite wide region. Therefore, it has been selected for the subsequent analysis.

\subsection{Proposed loop filter}

The modified LS estimator has considerably reduced the magnitude of the channel variations in the phase detector output. Additional attenuations can be introduced before supplying the interpolator with the timing adjustment values. Two equivalent methods can be employed to achieve this objective. The first one is to estimate the most important harmonics of phase error signal, for example, by means of the Goertzel algorithm, and to cancel them before entering the NCO. The second one is to eliminate these harmonics by placing notch filters in the loop. Both strategies offer equal

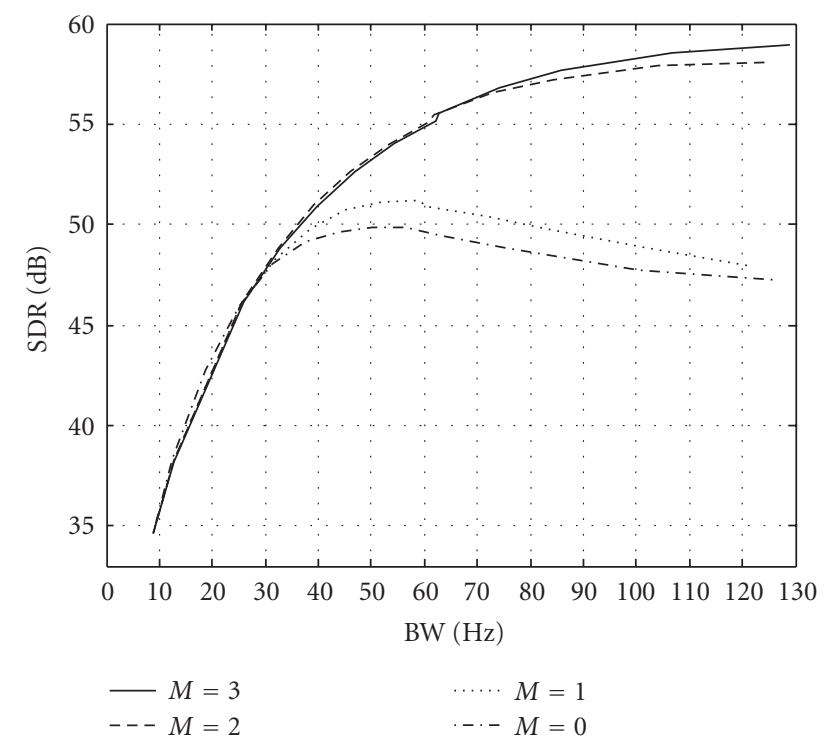

FIGURE 11: SDR obtained with the modified LS estimator for several numbers of notch filters.

performance, but due to the easier stability analysis, the latter one has been selected.

The modified loop filter is given by

$$
L(z)=\left(\alpha+\frac{\beta}{1-z^{-1}}\right) \prod_{i=1}^{M} H_{i}(z),
$$

where $H_{i}(z)$ are the transfer functions of notch filters obtained by applying the bilinear transform to the continuoustime second-order prototypes

$$
H_{i}(s)=\frac{s^{2}+\omega_{z, i}^{2}}{s^{2}+2 \xi_{i} \omega_{0, i} s+\omega_{0, i}^{2}},
$$

with $\omega_{z, i}=100 \pi i$. Up to three notch filters $(M=3)$ are employed in this study. Filter parameters have been heuristically selected to minimize the unavoidable resonance that appears in the passband and to achieve narrowband notches. As a result, $\xi_{i}$ is fixed to 0.1 in all filters and $\omega_{0, i}=\omega_{z, i} k(\alpha)$, where

$$
k(\alpha)= \begin{cases}1, & 0<\alpha \leq 4 \cdot 10^{-3} \\ \sqrt{1.1}, & 4 \cdot 10^{-3}<\alpha \leq 6 \cdot 10^{-3} \\ \sqrt{1.2}, & 6 \cdot 10^{-3}<\alpha \leq 10^{-2}\end{cases}
$$

The introduction of the notch filters considerably reduces the stability range of the overall loop filter. By means of the Nichols chart [12] of the overall loop frequency response, it has been determined that for $M=3$, the loop is stable only for $0<\alpha<21.5 \cdot 10^{-3}$. However, for $\alpha>10^{-2}(\mathrm{BW}>130 \mathrm{~Hz})$ the enormous growing experienced by the resonances in the passband invalidates the resulting frequency response.

Performance obtained with the modified loop is firstly assessed in terms of the SDR. Figure 11 depicts the SDR values experienced by the last carrier in the example channel for 
different values of $M$ and a 20 picosecond oscillator jitter. The conventional loop, $M=0$, has been included as a reference. As observed, gains obtained by using just one notch filter are rather small, while considerable improvement is obtained for $M=2$. This is due to the $100 \mathrm{~Hz}$ periodicity exhibited by the example channel. It is worth noting that the introduction of the notch filters eases the selection of the loop bandwidth, since the SDR is monotonically increasing for $M \geq 2$ in the selected range. Although not shown in Figure 11, small gains are obtained for $M>3$, specially in the apartment channel, in which more than $90 \%$ of the system carriers experience less than $150 \mathrm{~Hz}$ of Doppler spread [4].

Bit-rates corresponding to $M=3$ and different oscillator jitters are shown in Figure 12. Values have been computed using the SNDR and a lookup table. The new timing recovery scheme provides remarkable gains with respect to the conventional one (see Figure 9). The maximum bit-rate values obtained with the new system are, at least, 13\% higher than those obtained with the latter. This gain goes up to $24.5 \%$ when the oscillator jitter is 20 picoseconds and a fixed modulation strategy is employed. In addition, the bandwidth selection problem is now easier, since performance is less sensitive to this parameter.

Statistical values of the bit-rate loss computed over the 24 measured channels are now given. The procedure employed for the calculation is analogous to the one employed with the conventional timing recovery scheme. Results are shown in Table 2. As observed, performance degradation is considerably reduced in the apartment channels, especially when an adaptive modulation strategy is used, and is practically eliminated in the detached house ones. These significant improvements are particularly interesting when the reduced increment in the computational load is taken into account. Thus, the steady-state complexity of the modified LS estimator is equivalent to that of the conventional one, and the six-order filtering of the modified loop is performed only at symbol rate.

\section{CONCLUSIONS}

In this paper, the performance of a conventional DMT timing recovery scheme designed for LTI channels has been assessed when employed over indoor power lines.

The two main causes that limit the performance of the conventional strategy have been identified. One is the periodical bias introduced by the channel time variations in the phase error estimates. The other is the timing jitter introduced in the analog-to-digital conversion process. The latter, which is neglected in most studies, has revealed to be of particular importance in this case. It has been shown that optimal parameterization of the conventional scheme results from the tradeoff between attenuation of the cyclic bias and tracking capacity of the loop. Simulations carried out in a set of measured channels have demonstrated that this synchronization scheme limits the system bit-rate in many residential channels.

Two modifications have been proposed to overcome these shortcomings. The first is a new phase error estimator that takes into account the magnitude of the cyclic changes

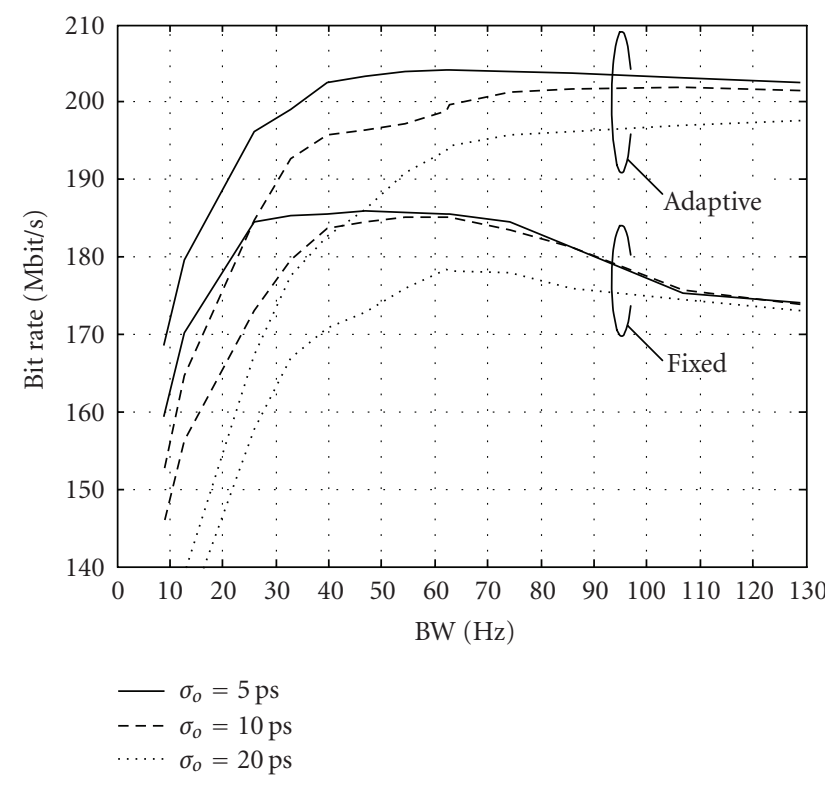

FIGURE 12: Bit-rate values obtained with the modified LS estimator and $M=3$.

TABle 2: Average bit-rate loss (\%) in each scenario with the modified LS estimator and $M=3$ when using the optimum loop bandwidth (on average).

\begin{tabular}{l|ccc}
\hline Scenario & $\sigma_{o}=20 \mathrm{ps}$ & $\sigma_{o}=10 \mathrm{ps}$ & $\sigma_{o}=5 \mathrm{ps}$ \\
\hline Apartment (adaptive) & 1.9 & 1.1 & 0.6 \\
Apartment (fixed) & 4.6 & 3.7 & 3.6 \\
Detached house (adaptive) & 0.1 & 0.1 & $<0.05$ \\
Detached house (fixed) & 0.1 & 0.1 & $<0.05$ \\
\hline
\end{tabular}

in the channel response. The second is the introduction of notch filters in the loop. This allows to reduce the periodical errors in the timing correction signal while retaining the loop ability to follow the jitter. Simulations confirm that performance degradation caused by the proposed scheme is negligible in most situations.

\section{ACKNOWLEDGMENT}

This work has been supported in part by the Spanish Ministry of Educación y Ciencia under CICYT Project no. TIC2003-06842.

\section{REFERENCES}

[1] TS 101867 V1.1.1, "Powerline Telecommunications (PLT); Coexistence of Access and In-House Powerline Systems," ETSI 2000.

[2] H. Philipps, "Performance measurements of power-line channels at high frequencies," in Proceedings of International Symposium on Power-Line Communications and Its Applications (ISPLCA '98), pp. 229-237, Tokyo, Japan, March 1998.

[3] F. J. Cañete, J. A. Cortés, L. Díez, and J. T. Entrambasaguas, "Modeling and evaluation of the indoor power line transmission medium," IEEE Communications Magazine, vol. 41, no. 4, pp. 41-47, 2003. 
[4] F. J. Cañete, J. A. Cortés, L. Díez, and J. T. Entrambasaguas, "Analysis of the cyclic short-term variation of indoor power line channels," IEEE Journal on Selected Areas in Communications, vol. 24, no. 7, pp. 1327-1338, 2006.

[5] T. Pollet and M. Peeters, "Synchronization with DMT modulation," IEEE Communications Magazine, vol. 37, no. 4, pp. 80-86, 1999.

[6] T. N. Zogakis and J. M. Cioffi, "The effect of timing jitter on the performance of a discrete multitone system," IEEE Transactions on Communications, vol. 44, no. 7, pp. 799-808, 1996.

[7] E. Martos, J. López, L. Díez, M. C. Aguayo, and J. T. Entrambasaguas, "Optimized interpolator filters for timing error correction in DMT systems for xDSL applications," IEEE Journal on Selected Areas in Communications, vol. 19, no. 12, pp. 24772485, 2001.

[8] J. A. Cortés, L. Díez, E. Martos, F. J. Cañete, and J. T. Entrambasaguas, "Analysis of timing recovery schemes for DMT systems over indoor power-line channels," in Proceedings of the 49th Annual IEEE Global Telecommunications Conference (GLOBECOM '06), San Francisco, Calif, USA, NovemberDecember 2006.

[9] T. Pollet, M. Van Bladel, and M. Moeneclaey, "BER sensitivity of OFDM systems to carrier frequency offset and Wiener phase noise," IEEE Transactions on Communications, vol. 43, no. 2-4, pp. 191-193, 1995.

[10] R. H. Walden, "Analog-to-digital converter survey and analysis," IEEE Journal on Selected Areas in Communications, vol. 17, no. 4, pp. 539-550, 1999.

[11] H. Kopmann, "A generalised parametric error model of ultrawideband analogue-to-digital conversion," in Proceedings of the 3rd Karlsruhe Workshop on Software Radios, pp. 101-110, Karlsruhe, Germany, March 2004.

[12] F. M. Gardner, Phaselock Techniques, John Wiley \& Sons, New York, NY, USA, 3rd edition, 2005.

[13] IEEE 1139-1999, "Standard Definitions of Physical Quantities for Fundamental Frequency and Time Metrology-Random Instabilities," IEEE 1999.

[14] A. Demir, A. Mehrotra, and J. Roychowdhury, "Phase noise in oscillators: a unifying theory and numerical methods for characterization," IEEE Transactions on Circuits and Systems I: Fundamental Theory and Applications, vol. 47, no. 5, pp. 655$674,2000$.

[15] E. A. Lee and D. G. Messerschmitt, Digital Communication, Kluwer Academic Publishers, Norwell, Mass, USA, 2nd edition, 1994.

[16] J. G. Proakis and M. Salehi, Communication Systems Engineering, Prentice-Hall, Upper Saddle River, NJ, USA, 1994.

José Antonio Cortés received the M.S. degree in telecommunication engineering from the University of Málaga (Spain) in 1998. In 1998, he received a fellowship from the R\&D Department of Alcatel Citesa (Málaga). In 1999, he worked for Alcatel España R\&D department (Madrid). At the same year, he joined the Communication Engineering Department of the University of Málaga, where he is currently working to-

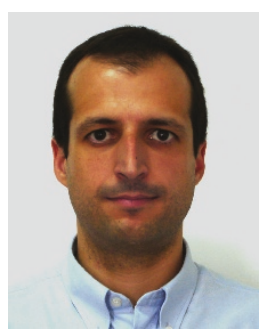
wards the Ph.D. degree. From 2000 to 2002, he collaborated with the Nokia System Competence Team in Málaga. His research interests include digital signal processing for communications, mainly focusing on synchronization and transmission techniques for highspeed power-line communications.
Luis Díez received the M.S. and Ph.D. degrees from the Polytechnic University of Madrid, Spain, in 1989 and 1995, respectively, both in telecommunications engineering. In 1984, he joined Fujitsu-España R\&D Center, where he worked in the development of modems. From 1987 to 1997, he was with the Department of Signals, Systems and Radiocommunication, Polytechnic University of Madrid. Since 1997, he has been with the Communication Engineering Department, University of Málaga. His research interests include digital communication, a field in which he has worked for many years. His experience includes most of its applications, that is, voice band, DSL and cable modems, satellite, mobile, and power-line communications, and so forth, as well as technical aspects, that is, synchronization, adaptive signal processing, modulation, coding, and multiple access.

Eduardo Martos received the M.S. and Ph.D. degrees in telecommunication engineering in 1996 and 2005, respectively, from the University of Málaga (Spain). In 1997 he joined the Communication Engineering Department, at University of Málaga, where he is currently an Associate Professor. His research activity includes digital signal processing for communications, primarily synchronization and channel estimation for PLC and xDSL systems, and performance analysis of wireless MIMO systems.

Francisco Javier Cañete received the M.S. and Ph.D. degrees in telecommunication engineering in 1996 and 2004, respectively, from the University of Málaga (Spain). In 1996, he worked for the Instrument and Control Department at the Empresa Nacional de Ingeniería y Tecnología (INITEC) in the design of power plants. In 1997, He worked for Alcatel España R\&D department in the design of wireless local loop systems.

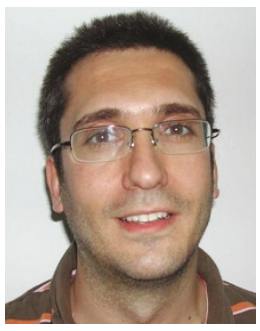
Since 1998, he has worked for the Communication Engineering Department, University of Málaga. From 2000 to 2001, he also collaborated with the Nokia System Competence Team in Málaga. His research activity is focused on digital signal processing for digital communications and his interests include channel modeling and transmission techniques for wireless systems and power-line communication systems.

José Tomás Entrambasaguas received the M.S. and Ph.D. degrees in 1975 and 1990, respectively, both from the Polytechnic University of Madrid, Spain. From 1975 to 1978, he worked at the Polytechnic University of Madrid. In 1978, he joined FujitsuEspaña R\&D Center, where he worked in the development of packet switching systems for data networks (in collaboration with Telefónica de España), data transmis-

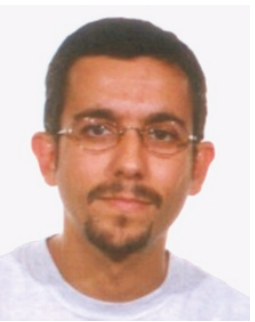
sion systems, and computer local area networks. In 1993, he joined the University of Málaga at the Communication Engineering Department, where his current interests include digital signal processing techniques applied to digital communication and methodologies for efficient and integrated development of complex communications systems. 